\title{
NORMAL SUBGROUPS OF THE MODULAR GROUP WHICH ARE NOT CONGRUENCE SUBGROUPS
}

\author{
MORRIS NEWMAN
}

Let $\Gamma$ be the $2 \times 2$ modular group of all linear fractional transformations

$$
\tau^{\prime}=\frac{a \tau+b}{c \tau+d}, \quad a d-b c=1,
$$

with rational integral coefficients $a, b, c, d$. For each positive integer $n$ let $\Gamma(n)$ denote the principal congruence subgroup of $\Gamma$ of level $n$, consisting of all substitutions (1) satisfying

$$
a \equiv d \equiv \pm 1(\bmod n), \quad b \equiv c \equiv 0(\bmod n) .
$$

A subgroup of $\Gamma$ containing a principal congruence subgroup $\Gamma(n)$ is said to be a congruence subgroup, and is of level $n$ if $n$ is the least such integer.

In a recent article [2] the writer determined all normal subgroups of $\Gamma$ of genus 1 (see [1] for the definition of the genus of a subgroup of $\Gamma$ ).

An interesting question that arises is to decide which of these are also congruence subgroups. In this note we show that there are just 4 such groups. This furnishes a new family of normal subgroups of finite index in the modular group which are not congruence groups (see [3] for other examples). The proof makes use of some recent work of $\mathrm{K}$. Wohlfahrt [4] on the definition of level for an arbitrary subgroup of finite index in $\Gamma$.

We let $x$ stand for the substitution

$$
\tau^{\prime}=-\frac{1}{\tau}
$$

and $y$ for the substitution

$$
\tau^{\prime}=-\frac{1}{\tau+1}
$$

Then $x^{2}=y^{3}=1$, and $\Gamma$ is the free product of the cyclic group of order 2 generated by $x$ and the cyclic group of order 3 generated by $y$.

The commutator subgroup $\Gamma^{\prime}$ of $\Gamma$ is a free group of rank 2 and of index 6 in $\Gamma$. $\Gamma^{\prime}$ is freely generated by the elements

Received by the editors May 18, 1964. 


$$
a=x y x y^{2}, \quad b=x y^{2} x y .
$$

If $w$ is any word of $\Gamma^{\prime}$, let $e_{a}(w), e_{b}(w)$ denote the sum of the exponents of $a$ and the sum of the exponents of $b$, respectively. Let $\Gamma^{\prime \prime}$ denote the second commutator subgroup of $\Gamma$, so that $\Gamma^{\prime \prime}$ consists of all words $w$ of $\Gamma^{\prime}$ such that $e_{a}(w)=e_{b}(w)=0$. It is shown in [2] that if $G$ is a normal subgroup of $\Gamma$ of genus 1 then $\Gamma^{\prime} \supset G \supset \Gamma^{\prime \prime}$, and that integers $p, m, d$ exist such that

$$
p>0, \quad 0 \leqq m \leqq d-1, \quad m^{2}+m+1 \equiv 0(\bmod d) ;
$$

and such that $G$ consists of those words $w$ of $\Gamma^{\prime}$ satisfying

$$
e_{a}(w) \equiv 0(\bmod p), \quad e_{b}(w) \equiv m e_{a}(w)(\bmod d p) .
$$

Conversely conditions (2) and (3) define a normal subgroup of $\Gamma$ of genus 1 . We denote $G$ by $(p, m, d)$.

Then we shall prove

THEOREM. The group $(p, m, d)$ is a congruence subgroup if and only if

$$
(p, m, d)=(1,0,1),(1,1,3),(2,0,1),(2,1,3) .
$$

Proof. In [4] Wohlfahrt defines the level of an arbitrary subgroup of finite index in $\Gamma$ as the least common multiple of the amplitudes of the parabolic vertices of the fundamental region of the group (see Wohlfahrt's paper for the definitions of these terms), and proves that for a congruence subgroup the two definitions of level coincide. It follows that if $G$ is a normal subgroup of finite index in $\Gamma$ and if $n=n(G)$ is the least positive integer such that the substitution $\tau^{\prime}=\tau+n$ is in $G$, then $G$ is of level $n$; and $G$ is a congruence subgroup if and only if $G \supset \Gamma(n)$.

It is shown in [2] that $n((p, m, d))=6$ for all groups $(p, m, d)$; and hence $(p, m, d)$ is a congruence subgroup if and only if $(p, m, d) \supset \Gamma(6)$. Since $(p, m, d)$ is of index $d p^{2}$ in $\Gamma^{\prime}$ and $\Gamma(6)$ of index 12 in $\Gamma^{\prime}$, it follows that $d p^{2} \mid 12$. All possible cases are now easily determined and the proof of the theorem is concluded.

\section{REFERENCES}

1. J. Lehner, Discontinuous groups and automorphic functions, Math. Surveys No. 8, Amer. Math. Soc., Providence, R.I., 1964.

2. M. Newman, $A$ complete description of the normal subgroups of genus one of the modular group, Amer. J. Math. 86 (1964), 17-24.

3. I. Reiner, Normal subgroups of the unimodular group, Illinois J. Math. 2 (1958), 142-144.

4. K. Wohlfahrt, An extension of F. Klein's level concebt, Illinois J. Math. 8 (1964), 529-535.

National Bureau of Standards 